\title{
BIFURCATION OF PERIODIC ORBITS ON MANIFOLDS, AND HAMILTONIAN SYSTEMS ${ }^{1}$
}

\author{
BY M. BOTTKOL \\ Communicated by R. T. Seeley, April 22, 1977
}

We consider a vector field $X_{0}$ having a whole submanifold $\Sigma \subset M$ of periodic points, and ask if any periodic orbits persist under small perturbation, i.e. do all vector fields $Y$ sufficiently near $X_{0}$ have periodic orbits lying near $\Sigma$. $\Sigma$ is assumed to be compact. Although in the general case there are simple counterexamples (e.g. on $\Sigma=n$ torus) some natural hypotheses on $\Sigma$ and the flow of $X_{0}$ guarantee periodic orbits for $Y$, which are thought of as bifurcating off the manifold $\Sigma$. Our method here is closely analogous to that of Moser [2], [3], and also his method of averaging on manifolds [1].

In the case of Hamiltonian flows, these methods take on added significance, and the classical action integral makes an appearance. Here the results may be viewed as an extension to $S^{1}$-actions of results of Weinstein carried out for $Z_{n}$. actions $[4],[5]$.

1. The general case. Let $X_{0}$ be a vector field on a manifold $M$ and $\phi^{t}$ its induced flow. A nondegenerate periodic manifold of $X_{0}$ of period $\tau$ is a $\phi^{t}$-invariant submanifold of $M$ such that $\phi^{\tau}(z)=z$ for all $z \in \Sigma$, and such that 1 is an eigenvalue of $d \phi_{z}^{\tau}$ of algebraic multiplicity $k=\operatorname{dim} \Sigma$.

We denote the space of vector fields over $M$ by $X(M)$, having the usual $C^{k}$ norm $\|\cdot\|_{k}$. We parametrize a neighborhood of the identity in $\operatorname{Diff}(M)$ by a neighborhood of $0 \in X(M)$ by taking a metric and setting $u(z)=\exp _{z} U(z)$, for $U \in X(M)$ small enough. We define an operator $P(u): X(M) \rightarrow X(M)$ which transports vectors at $z$ to vectors at $u(z)$ by setting, for $W \in T_{z} M$,

$$
P(u) W=\left.\frac{d}{d h}\right|_{h=0} \exp _{z}(U(z)+h W) .
$$

Lemma A. Let $X_{0}$ be a $C^{l+1}$ vector field on $M^{n}$ generating the flow $\phi^{t}$, having a compact nondegenerate periodic manifold $\Sigma$ of period 1. Suppose $Y$ is a vector field so that $\left\|Y-X_{0}\right\|_{l+1}<\epsilon$ in some neighborhood of $\Sigma$. Then for $\epsilon$ sufficiently small, there exists a $C^{l}$ vector field $V \in X(\Sigma), a C^{l}$ embedding $u$ : $\Sigma \rightarrow M$ near the inclusion, and $\phi^{t}$-invariant function $\lambda: \Sigma \rightarrow R$ so that

AMS (MOS) subject classifications (1970). Primary 34C25, 58F05, 34C30; Secondary $70 \mathrm{H} 99$.

${ }^{1}$ This work was partially supported by NSF Grant No. MCS74 03003 A 02. 
(i) $P(u) V(z)=d u_{z} X_{0}-\lambda(z) Y(u(z))$,

(ii) $\left[V, x_{0}\right]=0$,

(iii) $\left\langle V, X_{0}\right\rangle=0$ for $\langle\rangle , a \phi^{t}$-invariant metric on $\Sigma$. Writing $u(z)=\exp _{z} U(z), \mid z \in \Sigma$, let $U_{\Sigma}=$ component of $U$ tangential to $\Sigma$. Then $u$ and $V$ above are unique if we impose the normalization

(iv) $\int_{0}^{1} d \phi^{-t} U_{\Sigma}\left(\phi^{t}(z)\right) d t=0$.

In particular, if $V(\zeta)=0$, then $u\left(\phi^{t}(\zeta)\right)$ is a periodic trajectory of $Y$ of period $1 / \lambda(\zeta)$. The proof is to solve the linearized equations and then solve the nonlinear system by iteration. The transport operator $P(u)$ is needed not only to give equation (i) sense, but also its independence of the derivatives of $u$ is crucial to avoid loss of derivatives in the iteration.

COROLlaRY. If $X_{0}$ has a compact nondegenerate periodic manifold $\Sigma$ of period $\tau$, suppose that the flow $\phi^{t}$ of $X_{0}$ defines a free $S^{1}$ action on $\Sigma$. Then if the Euler characteristic $E\left(\Sigma / S^{1}\right) \neq 0$, every $Y$ sufficiently close to $X_{0}$ has at least 1 periodic orbit near $\Sigma$ with period close to $\tau$.

2. The Hamiltonian case. We now consider a manifold $P$ with a symplectic 2-form $\Omega$, and consider Hamiltonian vector fields $X_{0}, X$ with Hamiltonian functions $H_{0}, H$, which are close to one another. We consider a noncritical energy surface $E_{0}^{c}=\{z: H(z)=c\}$ of $H_{0}$ and the corresponding energy surface $E^{c}$ of $H$. We suppose that $X_{0}$ restricted to $E_{0}^{c}$ has a compact nondegenerate periodic manifold $\Sigma \subset E_{0}^{c}$. In order to compare the two flows, we take a diffeomorphism $\beta: E_{0}^{c} \rightarrow E^{c}$ between the two energy surfaces so that $\beta^{*} X=$ $d \beta^{-1} X(\beta(z))$ is a vector field on $E_{0}^{c}$ near $X_{0}$, and now apply Lemma $\mathrm{A}$ to get $u(z), V(z)$ and $\lambda(z)$ satisfying (a) $d u X_{0}-\lambda \beta^{*} X=P(u) V$, (b) $\left[V, X_{0}\right]=0$, (c) $\left\langle V, X_{0}\right\rangle=0$.

Assuming that $\Omega$ induces an exact 2-form $d \alpha$ in a neighborhood of $\Sigma$, set $\gamma_{t}=\beta \cdot u \cdot \phi^{t}(\zeta)$ and introduce the $X_{0}$-invariant function $S(\zeta)=\int_{\gamma_{t}(\zeta)} \alpha$ defined on $\Sigma$. $S(\zeta)$ is simply the classical "action" of the closed path $\gamma_{t}(\zeta)$ on $E^{c}$. Using (a) and (b), one finds $\left.Q V\right\lrcorner \Omega=d S$, where $Q: T M \rightarrow T M$ is a nonsingular map near the identity. $V$ is "almost" the Hamiltonian vector field of the function $S$, and $V(\zeta)=0$ if and only if $\zeta$ is a critical point of $S$.

One can replace the hypothesis that $\Omega=d \alpha$ with the assumption that $\Sigma$ is "exact" in the sense of Weinstein [5], [6].

In summary, the number of periodic orbits which persist on a given energy surface under Hamiltonian perturbations can be estimated by

$$
\text { \# critical points of } S \geqslant \text { Cat } \Sigma
$$

where "Cat" is the Liusternik-Schnirelman category. However, since $S$ is invariant under $S^{1}$-action on $\Sigma$ induced by $X_{0}$, the number of critical points of $S$ can be estimated in terms of the category at the quotient space $\Sigma / S^{1}$; see Weinstein [6]. 


\section{REFERENCES}

1. J. Moser, Regularization of Kepler's problem and the averaging method on a manifold, Comm. Pure Appl. Math. 23 (1970), 609-636.

2. A theorem of Weinstein and bifurcation theory, Univ. Catholique de Louvain, Inst: de Math. Pure et Appl. Rapport No. 61.

3. - Periodic orbits near an equilibrium and a theorem of Alan Weinstein, Comm. Pure Appl. Math. 29 (1976), 727-747.

4. A. Weinstein, Lagrangian manifolds and Hamiltonian systems, Ann. of. Math. (2) 98 (1973), 377-410.

5. - Normal modes for Hamiltonian systems, Invent. Math. 20 (1973), 47-57.

6. Symplectic V-manifolds, periodic orbits of Hamiltonian systems, and the volume of certain Riemannian manifolds, Comm. Pure Appl. Math. 30 (1977), 265-271.

COURANT INSTITUTE OF MATHEMATICS, NEW YORK UNIVERSITY, COURANT INSTITUTE, NEW YORK, NEW YORK 10012 